\title{
Efecto del fraccionamiento de la dieta y cantidad de hidratos de carbono en el control metabólico en pacientes con diabetes mellitus tipo 2, sin terapia con insulina
}

\author{
TAMARA LEIVA ${ }^{\mathrm{a}}$, KAREN BASFI-FER ${ }^{\mathrm{b}}$, PAMELA ROJAS, \\ FERNANDO CARRASCO, MANUEL RUZ O. ${ }^{\circ}$
}

\section{Effect of meal frequency and carbohydrate intake on the metabolic control of patients with type 2 diabetes mellitus}

Background: Increasing meal frequency is commonly used in the clinical practice as part of the nutritional treatment of patients with type 2 Diabetes Mellitus (DM2), although its effect on metabolic control parameters is controversial. Aim: To evaluate the association of energy intake, meal frequency, and amount of carbohydrates with fasting plasma glucose and glycosylated hemoglobin in a group of patients with DM2 without insulin therapy. Material and Methods: Dietary intake was evaluated in 60 subjects with DM2 through threeday food records. The meal frequency was estimated establishing the main meal times considering snacks. Results: Meal frequency was $4.7 \pm 1.1$ times per day. There was a positive association between glycosylated and fasting blood glucose levels $(p<0.01)$. Meal frequency was associated with energy intake $(p<0.01)$. When meal frequency, available carbohydrates and energy intake, body mass index and fasting plasma glucose were analyzed in a multiple linear regression model, fasting blood glucose was the variable that best predicted changes in glycosylated hemoglobin (45.5\%). Meal frequency had no association with glycosylated hemoglobin. Conclusions: Meal frequency showed no association with metabolic control parameters in DM2 patients.

(Rev Med Chile 2016; 144: 1247-1253)

Key words: Diabetes Mellitus, Type 2; Dietary Carbohydrates; Meals, Carbohydrates.

hile ha vivido un acelerado proceso de transición epidemiológica y nutricional, donde existe predominio de enfermedades crónicas no transmisibles, entre ellas diabetes mellitus tipo 2 (DM2), hipertensión arterial y enfermedades cardiovasculares ${ }^{1}$. En la Encuesta Nacional de Salud del año 2010, la prevalencia de diabetes mellitus fue de 9,4\%2. La DM2 corresponde a $90-95 \%$ de los casos de diabetes mellitus ${ }^{3}$.

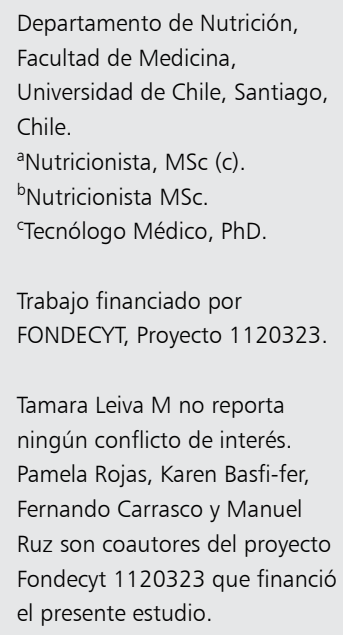

Tamara Leiva M no reporta ningún conflicto de interés. Pamela Rojas, Karen Basfi-fer, Fernando Carrasco y Manuel Ruz son coautores del proyecto Fondecyt 1120323 que financió el presente estudio.

Recibido el 29 de enero de 2016, aceptado el 31 de agosto de 2016

Correspondencia a:

Karen Basfi-fer O.

Av. Independencia 1027.

Correo 7, Santiago, Chile.

Fono: 229786770

kbasfifer@med.uchile.cl
La terapia nutricional es un componente clave en el manejo de esta condición, incluyendo, entre sus objetivos, tratar las complicaciones a corto y largo plazo asociadas a esta patología, ${ }^{3,4}$. En la literatura se ha observado que la terapia nutricional puede mejorar el control glicémico reduciendo los niveles de hemoglobina glicosilada (HbA1c) entre 1,0 y $2,0 \%$, donde la variable nutricional que ha probado tener el mayor impacto en el control 
metabólico es la modificación de la ingesta de hidratos de carbono ${ }^{3-5}$. Sobre la base de las recomendaciones de la American Diabetes Association (ADA) y Diabetes $U K$, la evidencia no es concluyente en relación a la cantidad de hidratos de carbono que debieran consumir los pacientes con DM2, sin embargo, propone monitorear su ingesta mediante el conteo de hidratos de carbono ${ }^{3,5}$. Por otra parte, las guías canadienses recomiendan una ingesta mínima de $45 \%$ del requerimiento energético diario en forma de hidratos de carbono, para reducir el riesgo de consumir dietas elevadas en grasas y aumentar el riesgo cardiovascular ${ }^{6}$.

En la práctica clínica es habitual recomendar a pacientes con DM2 fraccionar su ingesta en varias porciones, con el objetivo de regularizar el peso corporal y optimizar el control metabólico ${ }^{7}$. Las recomendaciones internacionales proponen fraccionar la alimentación en pacientes que necesiten bajar de peso y en aquellos con terapia insulínica, con el objetivo de coordinar la ingesta de carbohidratos con la dosis de insulina ${ }^{3}$. Los estudios realizados han mostrado cambios en el metabolismo de los hidratos de carbono en respuesta a un mayor fraccionamiento de la dieta, aumentando la tolerancia a la glucosa y las concentraciones de insulina ${ }^{4,8-10}$, sin embargo, un estudio sugiere que no habría diferencias significativas en la glicemia entre pacientes con una dieta fraccionada y pacientes con una dieta con menos tiempos de comida $^{11}$. La controversia sobre los resultados del fraccionamiento se puede asociar a diferencias metodológicas y a un relativo desinterés por investigar los efectos del fraccionamiento dietario sobre parámetros de control metabólico en pacientes con DM2, a pesar de ser utilizado recurrentemente como parte de las recomendaciones en la práctica clínica. Por lo anterior, el objetivo del presente estudio es relacionar la ingesta energética, el fraccionamiento de la dieta y la cantidad de hidratos de carbono totales con HbAlc y glicemia en ayuno, como parámetros de control metabólico, en un grupo de pacientes con DM2 sin terapia insulínica.

\section{Sujetos y Métodos}

\section{Sujetos}

Se evaluaron 60 sujetos con DM2 con menos de 10 años desde el diagnóstico, con un rango de edad entre 30 y 65 años, con un índice de masa corporal (IMC) entre 20 y $40 \mathrm{~kg} / \mathrm{m}^{2}$, variación de peso $<5 \%$, al menos por 3 meses antes del reclutamiento, hemoglobina glicada (HbAlc) $<9 \%$ o glicemia en ayunas $<180 \mathrm{mg} / \mathrm{dL}$. Fueron excluidos del estudio: sujetos con insulinoterapia, historia de cetoacidosis o síndrome hiperglucémico hiperosmolar no cetósico, con patología renal, hepática o accidente cerebrovascular.

Este estudio fue aprobado por el Comité de Bioética de la Facultad de Medicina de la Universidad de Chile y cada participante entregó su consentimiento escrito para participar.

\section{Antropometría}

Se midió la talla con un estadiómetro adosado a la balanza (con precisión de $0,1 \mathrm{~cm}$ ). El peso corporal se determinó con una balanza digital SECA ${ }^{\oplus}$ (Seca ${ }^{\circledR}$ 767, Hamburgo, Alemania. Precisión de $1 \mathrm{~mm}$ ). Las mediciones fueron hechas con los sujetos descalzos, peso distribuido uniformemente y vistiendo ropa ligera. Se calculó el índice de masa corporal (IMC) dividiendo el peso corporal por el cuadrado de la estatura, expresándolo en $\mathrm{kg} / \mathrm{m}^{212}$.

\section{Ingesta dietética}

Los pacientes completaron registros alimentarios de tres días: dos días de semana y uno de fin de semana, previo un entrenamiento por nutricionista. Se calculó el aporte de nutrientes de la dieta con el programa computacional Food Processor 2 (Food Processor II ${ }^{\circledR}$, ESHA Research, Salem, OR, USA), para evaluar y cuantificar la ingesta calórica y macronutrientes, con mayor énfasis en la cantidad de hidratos de carbono por tiempo de comida.

Se validaron los datos de la ingesta reportada calculando la razón ingesta de energía: gasto energético. La ingesta energética se estimó calculando el promedio de los tres días de registros alimentarios y el gasto energético se calculó utilizando la ecuación de Harris y Benedict agregando un factor de actividad física sedentaria de 1,3.

\section{Fraccionamiento}

Se contabilizó el número total de tiempos de comida realizados, y se establecieron los tiempos de comida principales: desayuno, almuerzo, once y cena. Se consideró cualquier alimento fuera de estos tiempos de comida como colaciones, las cuales se clasificaron según la hora de su consumo en colaciones AM (antes del mediodía) y colaciones PM (posterior al mediodía). 


\section{Exámenes de laboratorio}

En condiciones de ayuno se extrajo $30 \mathrm{~mL}$ de sangre de los sujetos estudiados para la determinación de hemoglobina glicada mediante HPLC (cromatografía líquida de alta precisión) y glicemia (método de hexoquinasa).

\section{Análisis estadístico}

Los análisis estadísticos se realizaron con el software SPSS (versión 22.0 SPSS Inc, Chicago, IL, USA) y se consideró el valor $\mathrm{p}<0,05$ para significación estadística. Se utilizó el test de Kolmogorov-Smirnov para determinar la distribución normal de los datos. Aquellas variables que mostraron normalidad se analizaron con el coeficiente de correlación de Pearson para establecer asociaciones, y se aplicó el test no paramétrico de Spearman para las variables que no mostraron normalidad en su distribución. Para correlaciones que no mostraron diferencias significativas con fraccionamiento (HbAlc, glicemia de ayuno e hidratos de carbono disponibles) se llevó a cabo una comparación entre primer y último tercil, analizada mediante pruebas $t$ de Student para muestras independientes.

Se aplicó el modelo de regresión lineal múltiple según método stepwise para evaluar los principales factores determinantes del control metabólico (fraccionamiento trabajado como variable continua, cantidad de hidratos de carbono por tiempo de comida, glicemia en ayuno). Para aquellas variables que no presentaron distribución normal se aplicó logaritmo natural.

\section{Resultados}

Las características generales de la muestra se describen en la Tabla 1 . Cabe mencionar que $45 \%$ de los sujetos presentaba un IMC considerado como sobrepeso, $36 \%$ como obesidad clase I y el restante $19 \%$ estaba en rangos de normalidad. La hemoglobina glicada mostró que 75\% de los sujetos tenía un buen control metabólico definido por una $\mathrm{HbAlc}<7 \%$.

La Tabla 2 muestra la ingesta de energía y macronutrientes calculada a partir de los tres registros de 24 h ( 2 días de semana y 1 día de fin de semana). La distribución de ingesta de energía según macronutrientes fue de: $18,8 \%$ como proteínas, $54,8 \%$ de hidratos de carbono y $26,4 \%$ de lípidos.
Al realizar la validación de la ingesta de energía reportada se observó un sub-reporte en $22 \%$ de la muestra, mientras que $46 \%$ de los sujetos realizó un reporte adecuado y $11,5 \%$ de la muestra sobre-reportó su ingesta energética en los registros alimentarios de tres días.

El fraccionamiento de la dieta fue de 4,7 $\pm 1,1$ tiempos de comida al día, con un mínimo de 3 y un máximo de 8 . Al analizar por porcentajes, 30\% de los sujetos realizaron 5 tiempos de comida, $27,8 \% 4$ tiempos de comida y $23 \%$ consumió en promedio 3 comidas al día. Todos los pacientes registraron consumo de desayuno y almuerzo, mientras que la once fue consumida en $85 \%$ de la muestra, la cena en $45 \%$ y las colaciones AM y PM mostraron un consumo de $72 \%$ y $62 \%$, respectivamente.

Se observó una asociación positiva y significativa entre HbA1c y glicemia de ayuno (Figura 1). Se encontró una asociación positiva $(\mathrm{p}<0,01$; r: 0,323 ) entre fraccionamiento e ingesta de energía, es decir, a mayor fraccionamiento, los sujetos aumentaban la ingesta energética (Figura 2). Cuando se analizó el fraccionamiento con $\mathrm{HbAlc}$, cantidad de hidratos de carbono disponibles y glicemia en ayuno no se encontraron asociaciones estadísticamente significativas.

Al compararar el tercil que fraccionó menos y el tercil que fraccionó más veces al día, no se observaron diferencias significativas en los valores de HbAlc, glicemia en ayuno y cantidad de hidratos de carbono disponibles. Asimismo, los hidratos de carbono disponibles no incidieron significativamente en los valores de glicemia de ayuno y HbAlc.

Tabla 1. Caracterización de la muestra

\begin{tabular}{|lc|}
\hline Variable & (n) Prom \pm DE \\
$\mathrm{n}$ & $(61)$ \\
\hline Edad (años) & $55,1 \pm 7,1$ \\
\hline Sexo (hombres/mujeres) & $(32 / 29)$ \\
\hline Peso $(\mathrm{kg})$ & $79,6 \pm 14,8$ \\
\hline Talla $(\mathrm{m})$ & $1,64 \pm 0,1$ \\
\hline IMC $\left(\mathrm{kg} / \mathrm{m}^{2}\right)$ & $29,4 \pm 3,6$ \\
\hline HbA1c $(\%)$ & $6,5 \pm 0,8$ \\
\hline Duración diabetes $(<5$ años $/ \geq 5$ años) & $(32 / 29)$ \\
\hline
\end{tabular}




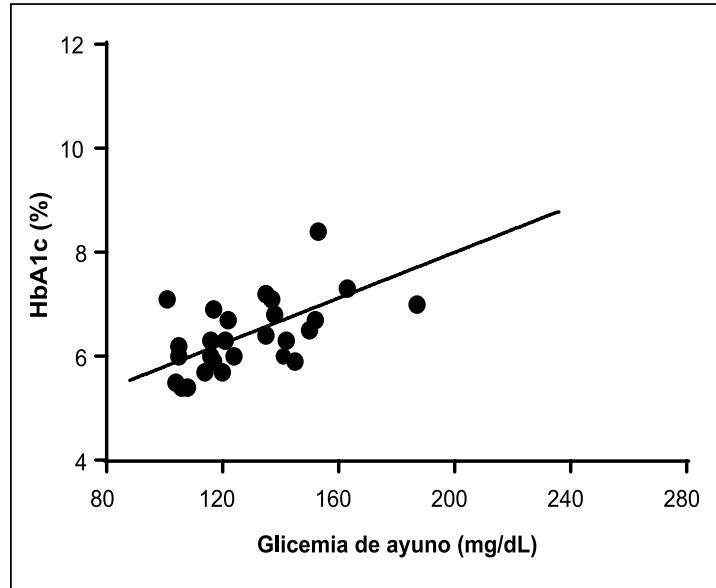

Figura 1. Asociación lineal entre $\mathrm{HbA} 1 \mathrm{c}$ y glicemia de ayuno. $R=0,67 ; p<0,001$.

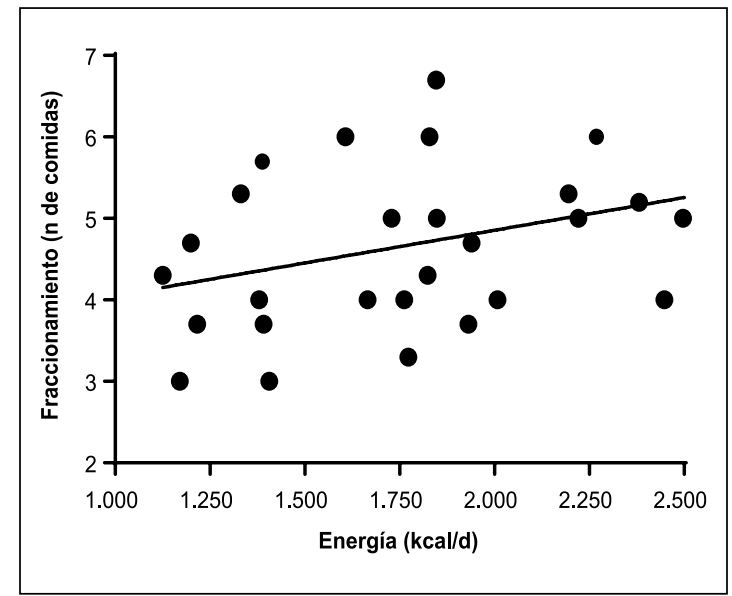

Figura 2. Asociación lineal entre fraccionamiento de la dieta e ingesta calórica. $R=0,33 ; p=0,01$.

Tabla 2 Distribución de ingesta de energía y macronutrientes

\begin{tabular}{|lccccc|}
\hline & Energía (kcal) & Proteínas (g) & CHO (g) & CHO disp. (g) & Lípidos(g) \\
\hline Total & $1.841,4 \pm 439,8$ & $83,1 \pm 29,4$ & $249,3 \pm 75,8$ & $227,7 \pm 74,2$ & $68,9 \pm 28,0$ \\
\hline Desayuno & $387,0 \pm 160,1$ & $23,5 \pm 29,3$ & $69,2 \pm 41,2$ & $63,7 \pm 45,2$ & $18,9 \pm 29,6$ \\
\hline Almuerzo & $638,6 \pm 195,3$ & $32,6 \pm 13,4$ & $68,6 \pm 32,8$ & $58,1 \pm 31,7$ & $26,5 \pm 8,2$ \\
\hline Once & $477,1 \pm 194,0$ & $18,4 \pm 10,0$ & $70,2 \pm 34$ & $65,2 \pm 30,1$ & $14,1 \pm 7,2$ \\
\hline Cena & $442,9 \pm 207,0$ & $22,1 \pm 11,3$ & $48,5 \pm 39,3$ & $43,7 \pm 38,2$ & $18,3 \pm 9,6$ \\
Colación AM & $167,4 \pm 156,3$ & $4,9 \pm 7,1$ & $29,7 \pm 22,8$ & $28,6 \pm 23,8$ & $3,7 \pm 7,0$ \\
Colación PM & $171,2 \pm 117,7$ & $3,3 \pm 1,9$ & $30,2 \pm 20,4$ & $27,8 \pm 9,4$ & $4,4 \pm 6,9$ \\
\hline
\end{tabular}

El fraccionamiento no influyó significativamente sobre la variabilidad de la HbAlc según el modelo de regresión lineal planteado, en el cual la glicemia de ayuno fue la variable que mejor predijo los cambios de la HbAlc (45,5\% de la variabilidad). La sumatoria total de los coeficientes de determinación de las variables analizadas pudo predecir 56\% de la variabilidad de los valores de HbAlc. El modelo de regresión lineal se puede observar en la Tabla 3.

\section{Discusión}

La importancia del fraccionamiento y la distribución de hidratos de carbono han sido tema
Tabla 3. Análisis de regresión múltiple: \%HbA1c

\begin{tabular}{|lcc|}
\hline \%HbA1c & $\begin{array}{c}\text { Coef. } \boldsymbol{\beta} \\
\text { estandarizado }\end{array}$ & $\mathbf{p}$ \\
\hline Constante & & 0,000 \\
Edad & 0,047 & 0,641 \\
Sexo & 0,225 & 0,032 \\
IMC & $-0,021$ & 0,827 \\
Energía & $-0,458$ & 0,009 \\
\hline Hidratos de carbono disponible & 0,226 & 0,146 \\
\hline Glicemia de ayuno & 0,600 & 0,000 \\
\hline Fraccionamiento & $-0,003$ & 0,979 \\
\hline
\end{tabular}

$R=0,75 ; R 2=0,56 ; p=0,000$. 
de debate en diferentes asociaciones internacionales, cuestionándose su utilidad y efectividad en el tratamiento del paciente con $\mathrm{DM} 2^{3}$. En las últimas actualizaciones, estas recomendaciones han perdido protagonismo; la ADA no presentó recomendaciones sobre fraccionamiento de la dieta para personas con DM2 no insulinorrequirientes, ya que falta evidencia de su real utilidad ${ }^{3}$. Por otro lado, las guías canadienses recomiendan mantener orden con los horarios de alimentación para mejorar el control glicémico en pacientes con $\mathrm{DM} 2^{6}$. A pesar de este contexto, el fraccionamiento y uso de colaciones, son prácticas que siguen vigentes y ampliamente utilizadas en la actualidad ${ }^{1}$.

El fraccionamiento se ha asociado con mejoras en el perfil lipídico, en las concentraciones de insulina y sensibilidad a insulina en sujetos sanos $y$ en sujetos con DM2 $2^{1,13}$, sin embargo, en este estudio el fraccionamiento no se asoció con un mejor control metabólico, ya que no contribuyó significativamente en los niveles de glicemia en ayuno y $\mathrm{HbAlc}$ de la muestra. $\mathrm{Al}$ respecto, Mekary y cols. observaron que sólo se produce una mejoría metabólica cuando, a pesar del fraccionamiento, la ingesta energética total se mantiene constante ${ }^{14}$. Lo anterior difiere de nuestros resultados, ya que se observó una relación positiva entre fraccionamiento e ingesta de energía, es decir, a mayor fraccionamiento, aumenta la ingesta energética diaria. Así, al perpetuar el balance energético positivo en este tipo de pacientes, puede contribuir a la ganancia de peso y a un peor control metabólico ${ }^{6}$.

Los estudios científicos que analizan los posibles efectos del fraccionamiento sobre el control metabólico proponen un diseño metodológico que incluye un fraccionamiento mayor respecto a una dieta habitual y amplia variabilidad en la distribución de energía y macronutrientes por tiempo de comida ${ }^{15-18}$, condiciones que poseen la desventaja de ser poco reproducibles en la cotidianidad como posible abordaje terapéutico del paciente con DM2. Esto deja de manifiesto la necesidad de más estudios que evalúen el fraccionamiento controlando la ingesta total de energía e hidratos de carbono.

En las indicaciones alimentarias, el fraccionamiento se traduce en tiempos de comida, al respecto, en este estudio hubo diferencias al compararlos con los resultados observados en la Encuesta Nacional de Consumo Alimentario $(\text { ENCA })^{19}$. El 100\% de los pacientes desayunaron y almorzaron, mientras que en la ENCA, 96\% de la muestra desayunó y $89,1 \%$ almorzó. La cena fue el tiempo de comida que presentó la mayor variación porcentual entre ambas mediciones $(22 \%)$, mientras que las colaciones, en general, registraron un mayor consumo, donde la ingesta de colación AM fue 47\% y la colación PM 29\%, por sobre el consumo nacional. Estas diferencias se pueden atribuir a una mayor adherencia a la consejería nutricional de integrar la cena y el consumo de colaciones a media mañana y media tarde para optimizar el control metabólico respecto a la población chilena general.

En la actualidad no hay consenso sobre la distribución óptima de calorías y macronutrientes para pacientes con DM2, pero su distribución debiera basarse en la evaluación individualizada de los patrones de alimentación actuales, preferencias personales y objetivos metabólicos ${ }^{3,6,10,15}$. Otras recomendaciones se orientan, además, a centrarse en la ingesta total de energía, en lugar de la distribución de las distintas fuentes de energía para el óptimo control glicémico ${ }^{4}$. La cantidad de hidratos de carbono es considerada un fuerte predictor de la respuesta glicémica, sumado al seguimiento de su ingesta como estrategia clave para alcanzar el control metabólico. Se recomienda que la ingesta mínima de hidratos de carbono supere $45 \%$ del valor calórico total o, en su defecto, un consumo mínimo de $150 \mathrm{~g}$ de hidratos de carbono diarios para garantizar la función cerebral, lo cual se cumple al analizar los datos de la muestra, donde se observó que el porcentaje del valor calórico total aportado por hidratos de carbono fue $56 \%$, con un consumo diario de $252,3 \pm 81,8 \mathrm{~g}^{1,9,20}$.

Con respecto al control metabólico, en este estudio los valores de glicemia en ayuno y el porcentaje de HbAlc se correlacionaron positiva y significativamente, mostrando que a medida que aumentan los niveles de glicemia en ayuno también aumentan los niveles de HbAlc, lo cual es esperado. Asimismo, se ha observado en la literatura que las hiperglicemias en ayuno aportan en mayor proporción en las variaciones de la HbAlc a medida que el control metabólico de la DM2 empeora ${ }^{3,5,6,21,22}$. Para determinar las principales variables que afectan la variación de la $\mathrm{HbAlc}$, se planteó un modelo de regresión lineal que incorporó las variables: fraccionamiento, glicemia de ayuno, ingesta de energía e hidratos de carbono, edad, índice de masa corporal y sexo. Este modelo 
explicó 56\% de la varianza, siendo los factores que mostraron coeficientes significativos la glicemia en ayuno, el IMC y la ingesta de energía. El fraccionamiento no mostró un efecto significativo, lo cual apoyaría aquellos estudios que asignan una importancia menor a este componente. Sin embargo, es importante señalar que $44 \%$ restante de la varianza no puede ser explicada por este modelo. Será de interés incluir otros factores, tales como la influencia de glicemias de diferentes horas del día u otras variables que no fueron considerados en este estudio y sus mecanismos de acción para contribuir a retrasar las complicaciones crónicas y mejorar la calidad de vida de los pacientes con DM2.

En resumen, no se encontró asociación entre el fraccionamiento e ingesta de hidratos de carbono con parámetros de control metabólico en los pacientes con diabetes tipo 2 estudiados, sin embargo, estos resultados podrían ser distintos si el fraccionamiento se acompañara de una ingesta calórica y de hidratos de carbono ajustadas a las necesidades de cada paciente y si no se hubiera asociado a una mayor ingesta de energía.

Agradecimientos: Financiado por FONDECYT, Proyecto 1120323.

\section{Referencias}

1. Minsal. Guías clínicas DM2. 2010. http://web.minsal.cl/ portal/url/item/72213ed52c3e23d1e04001011f011398. pdf (consultado el 10 de diciembre de 2015).

2. Encuesta Nacional de Salud ENS 2009-2010. Disponible en: http://web.minsal.cl/portal/url/item/bcb03d7bc28b64dfe040010165012d23.pdf (Consultado el 10 de diciembre de 2015).

3. American Diabetes Association. Standards of medical care in diabetes 2014. Diabetes Care 2014; 37 (S1): S1480.

4. Accurso A, Bernstein RK, Dahlqvist A, Draznin B, Feinman RD, Fine E, et al. Dietary carbohydrate restriction in type 2 diabetes mellitus and metabolic syndrome: time for a critical appraisal. Nutrition \& metabolism 2008; 5 (1): 9.

5. Dyson PA, Kelly TDT, Duncan A, Frost G, Harrison Zea. Diabetes UK evidence-based nutrition guidelines for the prevention and management of diabetes. Diabet Med 2011; 28 (11): 1282-8.

6. Canadian Diabetes Association Clinical Practice Guide- lines Expert Committee. Canadian Diabetes Association 2013 Clinical Practice Guidelines for the Prevention and Management of Diabetes in Canada. Can J Diabetes. 2013; 37 (1): 1499-2671.

7. Bertelsen J, Christiansen C, Thomsen C, Poulsen PL, Vestergaard S, Steinov A, et al. Effect of meal frequency on blood glucose, insulin, and free fatty acids in NIDDM subjects. Diabetes Care 1993; 16 (1): 4-7.

8. Jenkins D. Carbohydrate tolerance and food frequency. Bri J Nutr 1997; 77 (S1): S71-S81.

9. Leeanne MA, Ball MJ, Duncan AW, Mann J. Effect of isoenergetic intake of three or nine meals on plasma lipoproteins and glucose metabolism. Am J Clin Nutr 1993; 57 (3): 446-51.

10. Jenkins D, Ocana A, Jenkins A, Wolever TM, Vuksan V, Katzman L, et al. Metabolic advantages of spreading the nutrient load: effects of increased meal frequency in non-insulin-dependent diabetes. Am J Clin Nutr 1992; 50 (2): 461-7.

11. Jenkins D, Wolever T, Ocana T, Vuksan V, Cunnane S, Jenkins M, et al. Metabolic Effects of Reducing Rate of Glucose Ingestion by Single Bolus Versus Continuous Sipping. American Diabetes Association 1990; 39 (7): 775-81.

12. Gibson R. Principles of nutritional assesment. Second edition ed. New York: Oxford University press; 2005.

13. Arnold L, Mann JI, Ball MJ. Metabolic effects of alterations in meal frequency in type 2 diabetes. Diabetes Care 1997; 20 (11): 1651-4.

14. Mekary RA, Giovannucci E, Cahill L, Willet WC, van Dam RM, Hu FB. Eating patterns and type 2 diabetes risk in older women: breakfast consumption and eating frequency. Am J Clin Nutr 2013; 98 (2): 436-43.

15. García de Los Ríos M, Durruty AP, editors. Diabetes Mellitus. 3rd ed. Santiago de Chile: Mediterráneo; 2013.

16. Woerle HJ, Pimienta WP, Meyer C, Gosmanov NR, Szoke E, Szombathy T, et al. Diagnostic and therapeutic implications of relationships between fasting, 2-hour postchallenge plasma glucose and hemoglobin alc values. Arch Intern Med 2004; 164 (15): 9-23.

17. Monnier L, Lapinski H, Colette C. Contributions of fasting and postprandial plasma glucose increments to the overall diurnal hyperglycemia of type 2 diabetic patients. Diabetes Care 2003; 26 (3): 881-5.

18. The Diabetes and Nutrition Study Group (DNSG) of the European Association for the Study of Diabetes. Recommendations for the nutritional management of patients with diabetes mellitus. Eur J Clin Nutr 2000; 54 (4): 353-5.

19. Encuesta nacional de consumo alimentario. Informe 
final. Santiago de Chile: Facultad de Medicina, Universidad de Chile, Departamento de Nutrición, Escuela de Nutrición, Escuela de Salud Pública; 2014. http://web. minsal.cl/sites/default/files/ENCA-INFORME_FINAL. pdf (acceso 26 octubre 2016)

20. Rohlfing CL, Wiedmeyer HM, Little RR, England JD, Tennil A, Goldstein DE. Defining the relationship between plasma glucose and HbA1c. Diabetes Care 2002; 25 (2): 275-8.
21. Shadman Z, Khoshniat M, Poorsoltan N, Akhoundan M, Omidvar M, Larijani B, et al. Association of high carbohydrate versus high fat diet with glycated hemoglobin in high calorie consuming type 2 diabetics. J Diabetes Metab Disord 2013; 12: 27.

22. Franz MJ, Powers MA, Leontos C, Holzmeister LA, Kulkami K, Monk A, et al. The Evidence for Medical Nutrition Therapy for Type 1 and Type 2 Diabetes in Adults. J Am Diet Assoc 2010; 110 (12): 1852-89. 